Mit der alljährlichen Grippeimpfung schützen Sie sich und Ihr Umfeld vor einer Grippeerkrankung und deren Folgen. Jede Person, die geimpft ist, schränkt die Übertragung der Viren auf andere Personen ein und trägt so zu der sogenannten Herdenimmunität bei. Gemeinsam kann es uns gelingen, die Anzahl der durch die saisonale Grippe bedingten schweren Erkrankungen zu senken und so insbesondere die vulnerable Bevölkerung zu schützen.

\title{
Haben wir uns womöglich einfach an die Grippe gewöhnt?
}

Fabian Eglia, Linda Hadorn ${ }^{b}$

a Projektleiter Nationaler Grippeimpftag, Kollegium für Hausarztmedizin (KHM); ${ }^{b}$ wissenschaftliche Mitarbeiterin Abteilung Public Health, FMH

In der letzten Grippesaison 2017/18 führte die saisonale Grippe zu hochgerechnet 331000 grippebedingten Arztkonsultationen - so viele wie schon seit mehreren Jahren nicht mehr. Mittels Grippeimpfung könnten viele dieser Fälle und auch daraus folgende Spitalaufenthalte vermieden werden. Dies ist insofern relevant, da auch 2018 die Grippewelle in die Schweiz kommen wird. Trotzdem ist die Durchimpfungsrate in der Schweiz seit Jahren tief. Es fragt sich, woran dies liegt. Nachfolgend ein Erklärungsversuch.

\section{Mangelndes Wissen in der Bevölkerung}

In der Bevölkerung verwechseln viele Personen die saisonale Grippe mit einer (mehr oder weniger harmlosen) Erkältung. Ein Schnupfen mit Halsweh ist jedoch definitiv keine Grippe. Denn diese kennzeichnet sich vor allem durch einen akuten Beginn und hohes Fieber (über $39^{\circ} \mathrm{C}$ ). Sie fesselt betroffene Personen nicht selten mehr als eine Woche ans Bett. Hier gilt es im Rahmen der Gesundheitskompetenz jedes Einzelnen, Aufklärungsarbeit zu leisten. Wer nämlich eine harmlose Erkältung als Grippe erachtet, wird sich kaum impfen lassen, da die Symptome nicht als einschneidend genug empfunden werden.

\section{Fehlende Betroffenheit von Einzelpersonen}

Wer sich im Umfeld etwas umhört, der wird schnell mit der folgenden Frage konfrontiert: Weshalb sollte ich mich impfen lassen, wenn ich selber die Grippe in den letzten Jahren nie hatte? Auch in Bezug auf diesen
Aspekt ist Aufklärungsarbeit zwingend notwendig. Einerseits muss als zentrales Argument auf die Solidarität und die Herdenimmunität hingewiesen werden. Weiter gilt es, auch die Problematik der veränderten Viren aufzuzeigen. Diese Aufklärungsarbeit wird manchmal als Sisyphusarbeit empfunden, denn die Meinungen der betreffenden Personen sind meist bereits gefasst, und Veränderungen bzw. ein Hinterfragen der eigenen Haltung ist bekanntermassen nicht immer einfach. Zudem sind wir alle auch geprägt von

Weshalb sollte ich mich impfen lassen, wenn ich selber die Grippe in den letzten Jahren nie hatte?

einer Kultur, in der wir aufgewachsen sind. Ist die Grippeimpfung nicht Teil dieser Kultur, wird dies auch dazu führen, dass wir eine Grippeimpfung eher nicht in Betracht ziehen.

Doch gerade für Gesundheitsfachpersonen ist es wichtig, dass sie auch zukünftig versuchen, dem mangelnden Wissen zu begegnen. Dies, um im Sinne der För- 
derung der Gesundheitskompetenz Vorurteile und Unkenntnis schrittweise aufzulösen. Damit sind wir zurück bei der Sisyphusarbeit: Aussagen wie «ich habe wegen der Grippeimpfung überhaupt erst die Grippe eingefangen» oder «ich hatte gestern kurz die Grippe» muss mit einer längeren Erklärung und Fachwissen entgegnet werden. Diese Aufgabe zu erfüllen fällt einfacher, wenn die Kräfte gebündelt und vorhandene Synergien genutzt werden.

\section{Fehlende Bereitschaft zur Impfung}

Die im vorangegangenen Abschnitt erwähnte Aufgabe von Gesundheitsfachpersonen führt jedoch zu einem weiteren wiederkehrenden Thema: Auch bei Gesundheitsfachpersonen gibt es die mangelnde Impfbereitschaft. Sie stellt seit Jahren eine Herausforderung dar. Die Gründe sind auch hier vielfältiger Natur (und werden im Rahmen dieses Artikels nicht weiter erläutert).

Es stellt sich die Frage, wie damit umgegangen werden soll. Die Ansätze in der Schweiz und weltweit sind vielschichtig. Immer wieder wird die Einführung eines Impfzwanges diskutiert. Dieser ist aufgrund moralischer und ethischer Überlegungen bisher, wohl zu Recht, nicht eingeführt worden. Innovative Ansätze, die in den letzten Jahren vor allem im stationären Bereich umgesetzt wurden, versprechen weit mehr Potential. Doch auch hier läuft es am Ende auf eine

\section{Auch bei Gesundheitsfachpersonen gibt es die mangelnde Impfbereitschaft.}

fortwährende Sensibilisierung und kontinuierliche Information hinaus. Selbst wenn dies über die letzten Jahre wenig Erfolg gezeigt hat und die Durchimpfungsrate bei Gesundheitsfachpersonen weiterhin tief ist. Verbesserungspotential liegt im interprofessionellen Ansatz: In Bezug auf die Grippeprävention gilt es, die
Kräfte zu bündeln, Synergien zu nutzen und gute Ansätze breiter einzusetzen, damit erfolgreiche Projekte und Ideen nicht lokale Phänomene bleiben.

Seitens Ärzteschaft (primär Haus- und Kinderärzte) ist der Nationale Grippeimpftag seit 2004 ein Beitrag zur Grippeprävention, der auf die Initiative des Kollegiums für Hausarztmedizin (KHM) zurückgeht. Der diesjährige Grippeimpftag findet am Freitag, 9. November 2018, statt. Erstmals steht er unter dem Patronat der Verbindung der Schweizer Ärztinnen und Ärzte (FMH) und wird in Kooperation mit dem Schweizerischen Apothekerverband (pharmaSuisse) durchgeführt.

\section{Die Grippe ist auch im Jahr 2018 \\ keine harmlose Krankheit.}

Am Freitag, 9. November 2018, können sich alle impfinteressierten Personen, auch spontan, zu einem empfohlenen Pauschalpreis («all inclusive») von 30 Franken gegen die Grippe impfen lassen. Das Angebot besteht in

- allen teilnehmenden Arztpraxen für impfinteressierte Personen (nicht nur Patientinnen und Patienten). Die Adressliste der teilnehmenden Praxen ist auf der KHM-Website abrufbar: www.kollegium.ch;

- allen teilnehmenden Apotheken für impfinteressierte gesunde Erwachsene. Apotheken kennzeichnen ihre Teilnahme durch den Aushang der Plakate zum Nationalen Grippeimpftag. Apotheken, die grundsätzlich Impfungen anbieten, sind unter www.impfapotheke.ch abrufbar.

Die Grippe ist auch im Jahr 2018 keine harmlose Krankheit. Es wäre fatal, wenn wir uns mittlerweile einfach an ihren wiederkehrenden Rhythmus gewöhnt hätten. Wir alle gemeinsam sind in der Pflicht, unsere Arbeit zu leisten - sei es Aufklärung, Information oder das Hinterfragen der eigenen Haltung. Die Grippe ist kein Problem der anderen; Solidarität ist heute wichtiger denn je - auch, wenn es um die Grippe geht. 